\title{
One hyperheuristic approach to two timetabling problems in health care
}

\author{
Burak Bilgin - Peter Demeester • Mustafa \\ Misır • Wim Vancroonenburg • Greet \\ Vanden Berghe
}

Received: date / Accepted: date

\begin{abstract}
We propose one general hyperheuristic approach for addressing two timetabling problems in the health care domain: the patient admission scheduling and the nurse rostering problem. The complex combinatorial problem of patient admission scheduling has only recently been introduced to the research community. In addition to the benchmark instance that was recently introduced, we present six new benchmark instances. A comparison between the hyperheuristic and previously developed approaches reveals a significant outperformance of the new solution method. Nurse rostering, on the other hand, is a well studied health care operation research problem. Until now, not many nurse rostering benchmark instances existed. Only very recently, several nurse rostering data sets were introduced during the First Nurse Rostering Competition. We show that one hyperheuristic can tackle both health care timetabling problems with good results.
\end{abstract}

\section{Introduction}

Hyperheuristics were introduced to obtain solutions for problems in a 'good enough soon enough - cheap enough' fashion [6]. Compared to metaheuristics, hyperheuristics are high level search and optimization methods [6]. Instead of operating directly on

Burak Bilgin, Peter Demeester, Mustafa Misır, Wim Vancroonenburg, Greet Vanden Berghe KaHo Sint-Lieven

Information Technology

Gebroeders Desmetstraat 1

9000 Gent

Belgium

Tel.: +00-32-926586100

E-mail: \{Burak.Bilgin,Peter.Demeester,Mustafa.Misir,Wim.Vancroonenburg,Greet.VandenBerghe\}@kahosl.be

Peter Demeester, Greet Vanden Berghe

K.U. Leuven, Campus Kortrijk

Department of Computer Science

Etienne Sabbelaan 53

8500 Kortrijk

Belgium 
a set of candidate solutions, like metaheuristics do, they operate on a set of (meta-) heuristics. This property allows developers to easily deploy various heuristics at hand. Another objective of hyperheuristics research is to generate synergy between heuristics involved in the search, by making use of their strengths, avoiding their weaknesses, and taking advantage of their combined capabilities [6].

Most hyperheuristic approaches select every iteration a (low-level) heuristic according to a certain method, and a move according to a so-called move acceptance criterion. The selected heuristic will be applied in that iteration, and according to the move acceptance criterion, the proposed move will be executed or not. The heuristic selection mechanism as well as the move acceptance criterion can be based on (meta)-heuristics. Dowsland et al. 12, for example, present a hyperheuristic that uses tabu search as heuristic selection method and simulated annealing as move acceptance criterion. They deploy the approach to determine shipper sizes for storage and transportation. Kendall and Mohamad [18 introduce a great deluge metaheuristic as acceptance criterion in their hyperheuristic, and apply the resulting solution method to the channel assignment problem in cellular communication. Ayob and Kendall [1] utilize three different Monte Carlo strategies as acceptance criteria in a hyperheuristic to optimize component placement sequencing for multi head machine. Hyperheuristics have been also successfully applied to several health care problems. Tabu search has been used as a heuristic selection method to tackle timetabling and rostering problems including nurse rostering in [7]. Bilgin et al. 2] apply a hyperheuristic to generate rosters for nurses in a Belgian hospital.

In this paper we apply one general hyperheuristic approach to solve two health care timetabling problems. We report on experiments with different heuristic selection mechanisms and move acceptance criteria. Although the proposed approach is rather general and widely applicable, it obtains competitive results.

The first problem is the patient admission scheduling problem, that is gaining increasing attention in health care practice. Hospitals experience more and more pressure to maximize their bed occupancy and at the same time minimize the duration of each patient stay. These may result in poor conditions for the patients such as unplanned transfers from one room to another, assignments to rooms that do not match the patients' preference, etc. The goal of the patient admission scheduling problem is on the one hand increasing the patients' comfort while at the same time assisting the admission scheduler with the execution of his/her task. The problem involves a hospital with several organizational units, e.g. wards, and a number of patients with given arrival dates and expected departure dates. Patient assignments (to a particular bed in a room of a ward) are subject to constraints concerning the medical equipment in the room, the medical skills of the personnel who belong to that ward, the patient's room preference, etc. Although the need for improved efficiency is high, we notice that the problem has not yet attracted the interest of a large group of researchers yet. The bed assignment problem is described in detail in [11, in which one problem instance is introduced and solved with a token-ring tabu search approach and some metaheuristic variants. Integer programming has also been applied to solve the problem instance, but no optimal solution could be obtained even after a week of computation. That paper sets a benchmark that enables comparison and evaluation of new algorithms.

Nurse rostering is the second health care problem that is studied in this paper. It is the process of assigning nurses to shifts, taking into account coverage constraints, or personal and legal constraints. Coverage constraints express the number of nurses needed per shift and per day to satisfy the daily demand of the department. This 
number depends, amongst other things, on the bed capacity of the department. Typically, the coverage constraint is formulated as a number of nurses with a certain skill type that need to be present on a particular shift of a day. For example, a coverage constraint can express that for every early shift, five nurses should be present, while during the night shifts only 2 nurses should be available. Next to the general coverage constraint, also some legal constraints should be taken into account. Of course, these depend on the country. In Belgium, for example, there should be at least 11 hours of rest between two consecutive appearances. This is colloquially translated into the constraint that no nurse should be assigned to more than one shift per day. The coverage and the single shift per day constraint could be modelled as conditions that need to be satisfied in order to obtain a feasible solution. Nurse rostering is in contrast to the patient admission scheduling problem a well-studied problem in operation research (see Section 3.3 .

The contribution of this paper is one hyperheuristic approach to solve the patient admission scheduling problem and the nurse rostering problem. The outline of the paper is as follows. First, we present a detailed problem description of the patient admission scheduling problem in Section 2, and compare with other problems described in the literature. Following, we describe the nurse rostering problem in Section 3 , and review the corresponding literature. In Section 4, the hyperheuristic approach is introduced. The stress is both on the high level selection strategy and on the low level heuristics. We describe the experimental setup for both health care problems and discuss the results in Section 5 Regarding the patient admission scheduling problem, the results are compared against previously generated results for the same benchmark problems. Regarding the nurse rostering problem, we compare with the results obtained with an integer linear programming approach. In Section 6, we conclude and put forward some promising directions for future research.

\section{Patient admission scheduling}

\subsection{Problem description}

The objectives and constraints of the patient admission scheduling problem have been formalized after extensive discussions with decision makers in hospitals and with people who are informed about the hospital occupancy rates that are enforced by the government. The problem that we delineated for this research does not consider intensive care units nor day clinics. Also, we suppose that every patient is attributed an admission and discharge date in advance. In other words, we do not consider patients on waiting lists.

The basic elements of the problem at hand are patients, wards, rooms and timeslots. Associated with a patient are: his/her treatment requirements (in terms of nursing and medical equipment), gender, age category, room preference and the first day and the duration of his/her stay (this is called the length-of-stay of the patient). Similarly, some characteristics are related to rooms, e.g. existing medical equipment, the number of beds and the ward to which they belong. A ward defines the possible treatments that the rooms are equipped for. A time slot corresponds to a night. The occupation of one bed during one time slot by a patient is called a patient stay unit. The time horizon that we look at equals $T$, which corresponds to the set of all timeslots. We consider that the patients' stay durations over that period are known in advance and 
do not change during the stay. The model captures the knowledge of a decision maker at time 0 .

The objective is to optimize the overall patient assignment, i.e. satisfy the patients' preferences, while respecting all the hard constraints to the problem. Hard constraints need to be satisfied in any solution that the algorithm comes up with. The hard constraints are:

1. Maximum one patient per bed and time slot;

2. The admission and discharge dates are fixed. In other words, these dates cannot be changed by the algorithm;

3. For each time slot during the length-of-stay of a patient, s/he must be assigned to a bed;

The quality of a solution is determined by the soft constraints. Soft constraints are applied either to patients, or to rooms, to patients and rooms at the same time. The objective function is the weighted sum of all the violations of the soft constraints. The optimization problem is a minimization problem.

1. Patients in the same room-time slot should have the same gender;

2. The number of room transfers should be minimized;

3. The ward of the patient should satisfy the requirement of his/her pathology;

4. The room of the patient should satisfy the mandatory/preferred requirements of his/her pathology;

5. The room of the patient should satisfy the specialism of his/her pathology;

6. The room preference type of the patient should be satisfied.

\subsubsection{Mathematical model}

\section{Sets and constants definitions}

- Let $P$ be the set of all patients;

- Let $B$ be the set of all beds;

- Let $R$ be the set of all rooms;

- Let $T$ be the set of all timeslots;

- Each patient is either male or female:

$$
g_{p}=\left\{\begin{array}{l}
0 \text { if patient } \mathrm{p} \text { is female } \\
1 \text { if patient } \mathrm{p} \text { is male }
\end{array}\right.
$$

- Let $f_{r, t}$ be the number of female patients in room $r$ at time slot $t$, then

$$
f_{r, t}=\sum_{p \in P}\left(1-g_{p}\right) \cdot r s_{p, r, t}
$$

- Let $m_{r, t}$ be the number of male patients in room $r$ at time slot $t$, then

$$
m_{r, t}=\sum_{p \in P} g_{p} \cdot r s_{p, r, t}
$$


- Set of patient stays:

$$
P S_{p}=\left\{t \in T \mid t \geq t_{s, p} \wedge t<t_{s, p}+d_{p}\right\}
$$

where $t_{s, p}$ is the start day of the stay of patient $p$, and $d_{p}$ is the duration of the patient's stay;

- Let $r p_{p, r}$ be the total room penalty of the soft constraints 3, 4, 5, and 6 in case the patient $p$ is assigned to room $r$. These penalties are known in advance, since for every patient assigned to a room, one can calculate the corresponding penalty, based on the soft constraints $3,4,5$, and 6 .

\section{Decision variables}

- Bed schedule

$$
b s_{p, b, t}=\left\{\begin{array}{l}
1 \text { if patient } p \text { is assigned to bed } b \text { on timeslot } t \\
0 \text { otherwise }
\end{array}\right.
$$

- Room schedule

$$
r s_{p, r, t}=\left\{\begin{array}{l}
1 \text { if patient } \mathrm{p} \text { is assigned to room } \mathrm{r} \text { on timeslot } \mathrm{t} \\
0 \text { otherwise }
\end{array}\right.
$$

- Transfer

$$
t r_{p, t}=\left\{\begin{array}{l}
1 \text { if } t \in P S_{p} \wedge t+1 \in P S_{p} \wedge \exists r \in R \mid r s_{p, r, t} \neq r s_{p, r, t+1}, \\
0 \text { otherwise }
\end{array}\right.
$$

\section{Hard constraints}

$$
\forall b \in B, \forall t \in T, \sum_{p \in P} b s_{p, b, t} \leq 1
$$

Equation 1 corresponds to the first hard constraint.

$$
\forall p \in P, \forall t \in P S_{p}, \sum_{b \in B} b s_{p, b, t}=1
$$

Equation 2 corresponds to hard constraints 2 and 3 .

\section{Soft constraints}

- Gender penalties:

$$
G P=\sum_{r \in R} \sum_{t \in T} \min \left(f_{r, t}, m_{r, t}\right)
$$

Equation 3 corresponds to the first soft constraint.

- The total number of transfers:

$$
T R=\sum_{p \in P} \sum_{t \in T} t r_{p, t}
$$

Equation 4 corresponds to the second soft constraint. 
- Room penalty:

$$
R P=\sum_{p} \sum_{r} \sum_{t} r s_{p, r, t} r p_{p, r}
$$

Equation 5 corresponds to remaining soft constraints.

\section{Objective function}

$\operatorname{MinObj}=w_{g} \cdot G P+w_{t} \cdot T R+w_{r} \cdot R P$,

with $w_{g}$ the weight of the violation of the gender constraint, $w_{t}$ the weight of the violation of the transfer constraint, and $w_{r}$ the weight of the violation of the room constraints.

\subsection{Related literature}

We refer to Gemmel and Van Dierdonck 14 for a comprehensive study about the patient admission scheduling problem. It is noted that the problem should not be solved without taking surgery capacity and availability of nurses into account. Smith-Daniels et al. 24 too identify concerns that should be taken into account when optimizing bed occupancy. Similar problems to patient admission scheduling are addressed with mathematical programming [22, local search [15] and a multi agent system [20].

Vermeulen et al. 27 describe a CT-scan scheduling problem, which will - when solved to optimality - contribute to efficient patient scheduling. Central diagnostic resources such as CT-scans are often bottlenecks in a hospital environment and the goal is to minimize the patients' waiting time and to maximize the resources' utilization. Patients are scheduled according to their arrival order. For every patient a random free time slot is selected that fits into the patient's time window. The goal in the rehabilitation patient scheduling paper of Chien et al. [8] is similar: reduce the patients' waiting times and increase the equipment usage. Since some of the rehabilitation therapies require a specific order of execution, this problem can be seen as a hybrid shop scheduling problem in which the medical resources correspond to the machines and the patients to the jobs. Chien et al. solve the problem with a genetic algorithm.

Vermeulen et al. 28] apply agent technology is applied to solve the patient appointment exchanging problem. Patients are represented by agents that try to decrease the patients' waiting time. Initially, patients are assigned to a schedule that consists of several small appointments which are attributed a time slot. Each agent will try to exchange appointments with other agents in order to obtain a better schedule. An agent accepts an exchange only if it does not worsen its schedule. Vermeulen et al. 26] describe the on line scheduling of outpatients that have a combination of appointments in different wards. The goal is to optimize the appointments of the outpatients and the wards' efficiency. This is a complex problem since it needs a lot of collaboration between the wards of the hospital. Similar to 28, agent technology is applied to model the wards and the outpatients. The agents communicate with each other to find the best time slot for each party involved.

The objective of the problems presented in the papers above is to assign patients to timeslots in which they can be examined or diagnosed. The smallest timeslots are 15 minutes. In this paper; timeslots correspond to an overnight stay. However, the major difference between the problem described in this paper and the other problems in the literature, is that we are also confronted with the problem of consecutiveness. 
This means that patients who have been admitted to the hospital for multiple nights, should preferably be assigned to the same bed during their stay. This constraint adds considerable complexity to the problem.

Hutzschenreuter et al. 17] describe an agent-based patient admission scheduling application for a highly decentralized problem. In order to analyse and evaluate different policies concerning bed scheduling, what-if scenario's are applied. Patients are automatically assigned to beds with a brute-force optimizer. The time needed to assign patients to a small number of beds in a planning horizon of one year was 12.8 hours. No further details are given about the brute-force algorithm. From all the problems that we came across in the literature, it bears the closest resemblance to the one that we address in this paper.

\section{Nurse rostering}

\subsection{Problem description}

The specific nurse rostering problem that is described, modeled, and solved in this paper is the one that is the subject of the Nurse Rostering Competition [16. The goal of the organizers of this competition is to attract researchers from other disciplines, so that this problem is tackled with new approaches. Next, the competition organizers also want to close the gap between theory and practice and to stimulate debate in the timetabling community (see [16]). Competitions like this should be encouraged, since by introducing benchmark instances for nurse rostering different approaches can be compared in an objective manner. We are aware of some assumptions in the nurse rostering benchmarks that do not fully correspond to the real world situation. For example, the problems do not take any previous assignments into account. They also limit the number of assignments per nurse per day to one shift. Anyway, for investigating the performance of the hyperheuristic, they are perfectly suitable.

Nurse rostering is the process of assigning nurses to shifts taking into account the coverage, personal and legal constraints. A roster consists of several days, that are further divided into shifts. For every shift of a day, there is a corresponding number of required nurses. This is called the coverage. The coverage constraint is the first hard constraint of the problem. The other hard constraint is that nurses can only be assigned at most to one shift per day. Each nurse is assigned exactly one contract, which provides the following information:

- the maximum and minimum number of assignments per planning period and per nurse;

- the maximum and minimum number of consecutive working days;

- the maximum and minimum number of consecutive free days;

- the maximum number of consecutive working weekends;

- whether the nurse needs two free days after being assigned to a night shift;

- whether a nurse has to work all days of the weekend (so-called complete weekends);

- whether a nurse has to work the same shift types during a working weekend;

- which combination of shift types are unwanted. For example, do not assign a nurse to the late shift of the first day, the early shift of the second day and the late shift of the following day;

- the shifts and days on/off requests of the nurses. These are requests of the nurses (not) to be assigned on certain shifts or days. 
The elements of these contract can be considered as the soft constraints of the problem. For all data instances of the Nurse Rostering Competition the planning period is 4 weeks, which includes 4 weekends, and the number of shifts per days ranges from 4 to 5 .

For the full problem description of the Nurse Rostering Competition instances, we refer to 16 .

\subsection{Mathematical model}

The mathematical model for this specific nurse rostering problem is influenced by the one of Burke et al. 4]. For the interested reader we have added the complete mathematical model for this specific problem in the Appendix.

3.3 Related literature

TODO: Greet

\section{Solution methods}

\subsection{Overview}

A local search neighbourhood corresponds to the set of all candidate solutions that can be reached from one solution by carrying out a specific move. A local search algorithm searches for a good quality solution by traversing the neighbourhoods of a single candidate solution. Tabu search, for example, adds some memory to the local search by applying a finite tabu list. Elements of every accepted modification are added at the start of the tabu list replacing the oldest item in the tabu list. If the quality of the candidate solution is better than the previous best solution, the modification is executed. If the modification does not lead to a better solution, the best move is accepted if that is not prohibited by the tabu list. This mechanism helps escaping from local optima and forces the algorithm to explore new regions of the solution space. In 11 the patient admission scheduling problem is tackled with a tabu search algorithm hybridized with a token-ring approach. This mechanism deploys several neighbourhoods, and carries out the search by switching between them in a circular fashion.

Hyperheuristics are solution methods that deploy a set of heuristics. This way they are distinguished from the metaheuristics approaches that operate directly on the solution space. Several classes of hyperheuristics have been developed: 'heuristics to choose heuristics' 7 or 'heuristics to generate heuristics' 5 . In this work, we consider hyperheuristics that choose heuristics. This mechanism iteratively selects a heuristic and applies it to a single candidate solution. After the selection step, the resulting candidate solution is either accepted or rejected. The hyperheuristic framework that is used in this article is based on the one described in 23. It consists of two main parts: the heuristic selection mechanism and the move acceptance criterion.

- The heuristic selection mechanism selects every iteration one heuristic from the set of low-level heuristics. 
- The move acceptance criterion decides every iteration which move is accepted. Several well-known metaheuristic methods can be applied as a move acceptance criterion.

The hyperheuristic framework is the same for both health care problems. The followed approach for both problems only differs in the solution representation, the construction of the initial solution, and the low-level heuristics. These parts contain information specific to each of the problems.

\subsection{Modeling approach}

For both health care problems, we describe in the following sections the solution representation and the objective function.

\subsubsection{Patient admission scheduling}

Since the modeling approach of the patient admission scheduling problem is fully described elsewhere (see [1]), only a brief model description is provided. A solution is represented as a set of matrices, each representing a ward of the hospital. The rows of an individual matrix correspond to the available beds in the ward, while the columns correspond to the timeslots. In the solution representation, a patient assignment is represented as a contiguous set of patient stay parts. There are as many patient stay parts as the length-of-stay of the corresponding patient. By choosing this particular representation, violations of the first hard constraint (assigning more than one patient to a bed) are automatically excluded.

The initial solution is constructed such that all patients are assigned to beds as long as there are free beds available. This automatically leads to satisfaction of the last hard constraint. The second hard constraint will always be fulfilled by only allowing low level heuristics that do not violate this constraint (see Section 4.3.1).

\subsubsection{Nurse rostering}

A solution for the nurse rostering problem is modeled as a 0-1 matrix, in which the columns represent the shifts arranged per day, and the rows represent the nurses. A nurse is assigned to a shift on a particular day if the value of the corresponding matrix element is 1 . Although the initial solution is randomly constructed, the initialization algorithm makes sure that the solution is feasible. This means that the coverage is met and that no nurse works more than one shift in the same day. Feasibility is maintained during the subsequent search by only considering assignment moves within the same column. No assignment can be removed without making a new one within the column. This means that the coverage constraint and the single shift per day constraint will always be satisfied.

In both problem cases the evaluation of the soft constraints will be included in the objective function, which is the weighted sum of their violations. 
4.3 Low-level heuristics

The heuristics that are called by the hyperheuristic are inspired by the mechanism of tournament selection in genetic algorithms. At each iteration, the selected heuristic creates a number of moves and returns the move that results in the best value of the objective function. The number of moves considered per iteration is a parameter of the search algorithm that we call the tournament size.

\subsubsection{Patient admission scheduling}

The heuristics only consider moves that do not change the admission and the discharge dates of the patients:

1. Swap two patients' assignments in the same ward. The ward and the patients are selected randomly.

2. Swap two patients' assignments in different wards. The wards and the patients are selected randomly.

3. Transfer all the assignments of a patient to (an) empty bed(s) in another ward. The source and destination wards, the patient, and the destination bed(s) are selected randomly.

4. Transfer all the assignments of a patient to (an) empty bed(s) in the same ward. The ward, the patient, and the destination bed(s) are selected randomly.

The source and destination beds in the first and the second heuristic can either be empty or assigned to a patient.

\subsubsection{Nurse rostering}

We present a sample of the low-level heuristics in the hyper-heuristic approach: in all the low-level heuristics, the first step is to randomly select two nurses $n_{1}, n_{2},\left(n_{1} \neq\right.$ $n_{2}$, and $n_{1}, n_{2} \in N$, with $N$ the set of nurses)

- randomly select a subset $d \subset D$, with $D$ the set of all days:

$$
\operatorname{swap}\left(\operatorname{roster}\left(n_{1}, d\right), \operatorname{roster}\left(n_{2}, d\right)\right)
$$

- randomly select a subset $w \subset W$, with $W$ the set of all weekends:

$$
\operatorname{swap}\left(\operatorname{roster}\left(n_{1}, w\right), \operatorname{roster}\left(n_{2}, w\right)\right)
$$

- randomly select a subset $v \subset V$, with $V$ the set of all work days (or $V=D \backslash W$ ):

$$
\operatorname{swap}\left(\operatorname{roster}\left(n_{1}, v\right), \operatorname{roster}\left(n_{2}, v\right)\right)
$$

In fact, we have a dozen low-level heuristics, which are all based on these three abstract heuristics. For example, we use a heuristic that selects the even or odd weekends (or work weeks) of two randomly chosen nurse rosters. Another low-level heuristic swaps one, two, three, four or five (non-)contiguous days of two randomly chosen nurses.

\subsection{Hyperheuristic framework}

As mentioned in Section 4.1 the hyperheuristic framework that is employed in this paper consists of two main parts: a mechanism to select one of the low-level heuristics during the search, and a criterion to accept moves. 


\subsubsection{Heuristic selection method}

The heuristic selection methods are simple random, choice function, and a dynamic heuristic set strategy with simple random.

- Simple random randomly selects a heuristic from a list of heuristics at each iteration.

- Choice function 9 considers a number of criteria: the performance of each heuristic and each pair of heuristics when called consecutively, and the elapsed time since the last call of a heuristic. The choice function consists of a linear combination of the following components (see 25] for more details):

- $f_{1}\left(h_{j}\right)$, which is the current performance of every heuristic $h_{j}$. This is expressed as $f_{1}\left(h_{j}\right)=\sum_{n} \alpha^{n-1}\left(\frac{I_{n}\left(h_{j}\right)}{T_{n}\left(h_{j}\right)}\right)$, where $I_{n}\left(h_{j}\right)$ corresponds to the change in the evaluation function of the heuristic $h_{j}$, and $T_{n}\left(h_{j}\right)$ corresponds to the amount of CPU time taken in heuristic $h_{j}$, and where $\alpha \in[0,1]$.

- $f_{2}\left(h_{k}, h_{j}\right)$, which is the joint performance of couples of heuristics $\left(h_{k}, h_{j}\right)$. This is expressed as $f_{2}\left(h_{k}, h_{j}\right)=\sum_{n} \beta^{n-1}\left(\frac{I_{n}\left(h_{k}, h_{j}\right)}{T_{n}\left(h_{k}, h_{j}\right)}\right)$, where $I_{n}\left(h_{k}, h_{j}\right)$ is the change in the evaluation function since the last time heuristic $h_{j}$ was called immediately after heuristic $h_{k}$, and where $T_{n}\left(h_{k}, h_{j}\right)$ is the amount of CPU time taken since the last time heuristic $h_{j}$ was called immediately after heuristic $h_{k}$, with $\beta \in[0,1]$.

- $f_{3}\left(h_{j}\right)$ which is the elapsed time since the last execution of heuristic $h_{j}$.

The resulting choice function can then be expressed as $f\left(h_{j}\right)=\alpha f_{1}\left(h_{j}\right)+\beta f_{2}\left(h_{k}, h_{j}\right)+$ $\delta f_{3}\left(h_{j}\right)$.

- The motivation behind the dynamic heuristic set strategy with simple random

[21] approach is to determine the best heuristic subsets for the different phases of a search. Each phase refers to a predefined number of iterations in which the performance, based on performance metric, of the available $n$ heuristics is measured. The lesser performing heuristics are excluded for a number of phases. The number of phases for the exclusion process is called the tabu duration.

In this study, we employ the following performance metric:

$$
p_{i}=M_{1}(i) w_{1}+M_{2}(i) w_{2}+M_{3}(i) w_{3}
$$

- $M_{1}(i)$ denotes the total number of new best solutions found by the heuristic $i$,

- $M_{2}(i)$ denotes the fitness improvement per execution time during the current phase by the heuristic $i$,

- $M_{3}(i)$ denotes the total fitness improvement per execution time by the heuristic $i$.

$w_{1}, w_{2}$ and $w_{3}$ are the weights for each sub performance metric and their values are assigned as $w_{1} \gg w_{2} \gg w_{3}$. This way, some kind of priority between these three sub performance metrics is provided.

The tabu duration value and phase length are determined based on the size of the heuristic set. The tabu duration is $d=\sqrt{2 n}$ and the phase length is $p l=d * 1000$ iterations. At the end of each phase, each heuristic gets a quality index $(Q I)$ value which can range between 1 to $n$. The best performing heuristic gets $n^{\prime}$ which is the 
number of heuristics in the current heuristic subset and the worst one gets 1 as its $Q I$. The excluded heuristics are considered as heuristics with $Q I=1$. Then, the average of the $Q I \mathrm{~s}$ is calculated :

$$
a v g=\left\lfloor\left(\sum_{i}^{n} Q I_{i}\right) / n\right\rfloor
$$

The heuristics with $Q I<a v g$ are excluded from the heuristic set for $d$ number of phases.

\subsubsection{Move acceptance criteria}

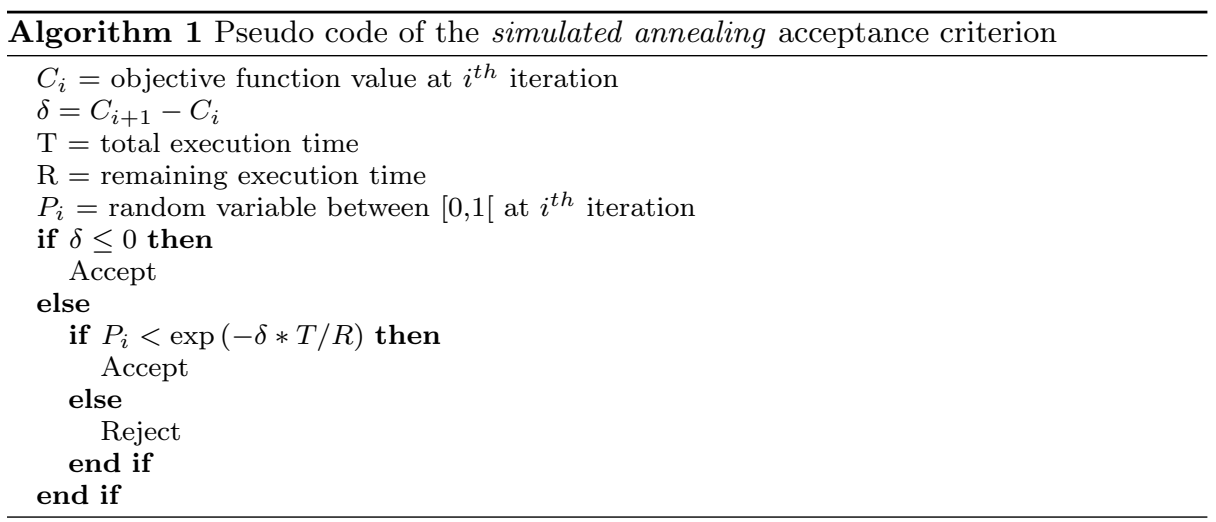

We have experimented with four acceptance criteria. The acceptance criteria are only improving, improving and equal, simulated annealing [19], and great deluge [13].

- Only the moves leading to solutions that are better than the current solution are accepted by the only improving acceptance criterion.

- The improving and equal acceptance criterion works similarly, except that it also accepts moves that result in solutions that are as good as the current solution.

- The simulated annealing algorithm accepts all improving and equal moves. The moves that result in solutions that are not at least as good as the current solution are carried out with a probability, which is decreased throughout the execution. This acceptance criterion is presented in Algorithm 1

- The great deluge acceptance criterion maintains a deluge level throughout the execution. The initial value of the deluge level is set equal to the cost value. Throughout the search, this level is reduced at each iteration. The criterion accepts all improving and equal moves and the moves that result in a cost value below the deluge level. This acceptance criterion is explained in Algorithm 2.

\section{Experiments}

All experiments were performed on Intel Core2Duo (3 GHz) PCs running Windows XP Professional SP3, with a Java 1.6 JRE configured to run in server mode with a heap size of $128 \mathrm{MB}$. 


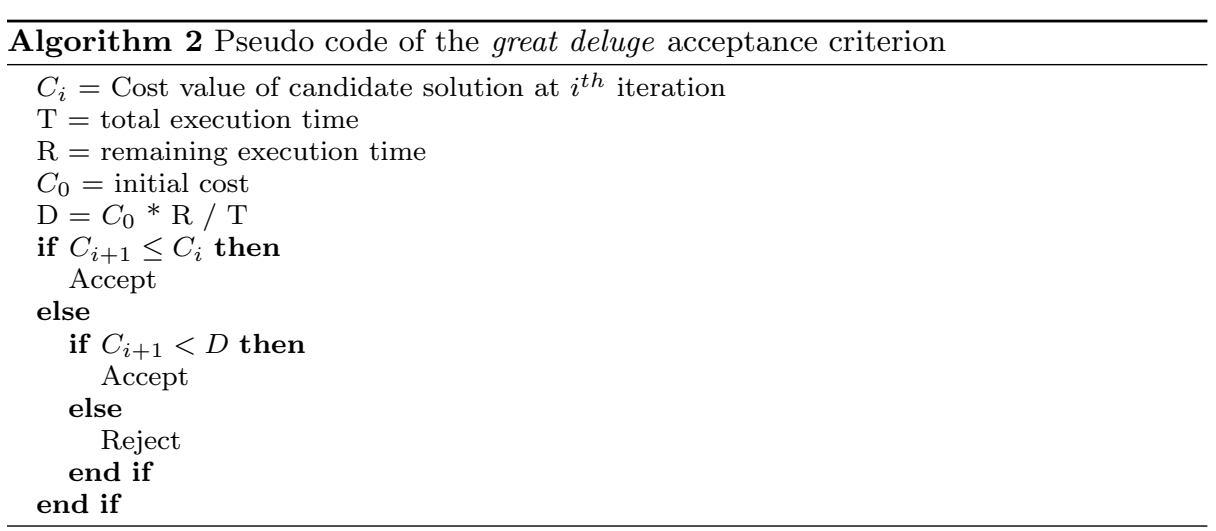

We experiment with the four different acceptance criteria: simulated annealing (SA), great deluge (GD), improving and equal (IE), and only improving (OI), and with the three heuristic selection mechanisms: simple random (SR), choice function $(\mathrm{CH})$, and the dynamic heuristic set strategy with simple random (DHS). Three different tournament sizes are explored: 4, 16, and 64. As a result, 36 hyperheuristic variations are tested on each patient admission scheduling and nurse rostering benchmark instance.

5.1 Patient admission scheduling problem

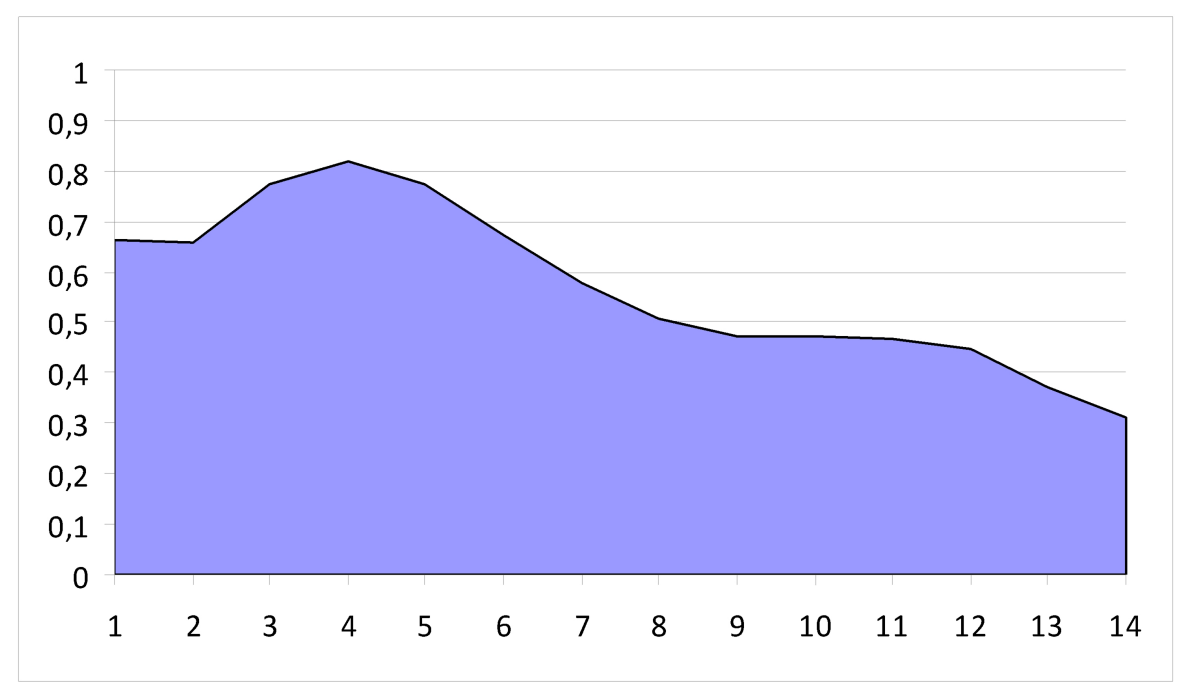

Fig. 1 Example of the occupancy rate over the planning horizon (benchmark instance 3).

The experiments are carried out on one existing and six new benchmark instances. The existing dataset (dataset 0) was first introduced in 11. All instances are auto- 


\begin{tabular}{|l||r|r|r|r|r|r|r|}
\hline Property / Benchmark Instances & $\mathbf{0}$ & $\mathbf{1}$ & $\mathbf{2}$ & $\mathbf{3}$ & $\mathbf{4}$ & $\mathbf{5}$ & $\mathbf{6}$ \\
\hline \hline Number of departments & 6 & 4 & 6 & 5 & 6 & 4 & 4 \\
\hline Number of rooms & 150 & 98 & 151 & 131 & 155 & 102 & 104 \\
\hline Number of beds & 447 & 286 & 465 & 395 & 471 & 325 & 313 \\
\hline Number of female patients & 339 & 315 & 359 & 365 & 361 & 279 & 349 \\
\hline Number of male patients & 321 & 337 & 396 & 343 & 385 & 308 & 336 \\
\hline Maximum occupancy & 0.69 & 0.77 & 0.79 & 0.82 & 0.75 & 0.73 & 0.91 \\
\hline Average occupancy & 0.50 & 0.60 & 0.60 & 0.57 & 0.54 & 0.49 & 0.64 \\
\hline
\end{tabular}

Table 1 The characteristics of the benchmark instances

matically generated based on several interviews with people responsible for scheduling patients in Belgium. Obtaining real-world data about patients turned out to be difficult, due to privacy issues. Although automatically generated, we are convinced that the datasets are realistic. Each benchmark instance has a planning horizon of two weeks. The properties of the benchmark instances are given in Table 1 For the new instances the length of stay of every patient (expressed in nights) is presented in Fig. $2(\mathrm{a}), 2(\mathrm{~b}), 2(\mathrm{c}), 2(\mathrm{~d}), 2(\mathrm{e}), 2(\mathrm{f})$ The occupancy rate varies over the planning horizon. The rate is higher during the week than in the weekend. The occupancy rate is also higher in the first week than in the second week. That resembles the real world situation in which patients' medical treatments are not always scheduled two weeks in advance. In Figure 1, the occupancy rate of benchmark instance 3 is depicted over the planning horizon as an example. In Table 2 the distribution of the room types is depicted. There are more quadruple rooms than double rooms, and more double rooms than single rooms. This is in correspondence with the situation in Belgian hospitals. The benchmark instances and the weights of the objective function can be found on the Patient Admission Scheduling website [10.

\begin{tabular}{|r|r|r|r|}
\hline & \% of single rooms & \% of double rooms & \% of quadruple rooms \\
\hline testdata 0 & $12 \%$ & $32 \%$ & $55 \%$ \\
\hline testdata 1 & $16 \%$ & $29 \%$ & $54 \%$ \\
\hline testdata 2 & $12 \%$ & $26 \%$ & $60 \%$ \\
\hline testdata 3 & $13 \%$ & $30 \%$ & $57 \%$ \\
\hline testdata 4 & $12 \%$ & $30 \%$ & $58 \%$ \\
\hline testdata 5 & $8 \%$ & $27 \%$ & $64 \%$ \\
\hline testdata 6 & $10 \%$ & $34 \%$ & $56 \%$ \\
\hline
\end{tabular}

Table 2 Distribution of single, double and quadruple rooms

In total, 36 different solution methods are tested for each of the patient admission scheduling benchmark instances. Each solution method is run 10 times for each benchmark instance. The termination criterion is the computation time, which is set to 3000 seconds for each run. In order to have an objective means of comparison with other researchers we opt to apply the same benchmark method as in the Second International Timetabling Competition ${ }^{1}$. They provide a benchmark program that calculates the maximum allowed computation time based on the specifications of each individual PC. We multiply the resulting computation time by 10 .

\footnotetext{
1 http://www.cs.qub.ac.uk/itc2007/index_files/benchmarking.htm
} 

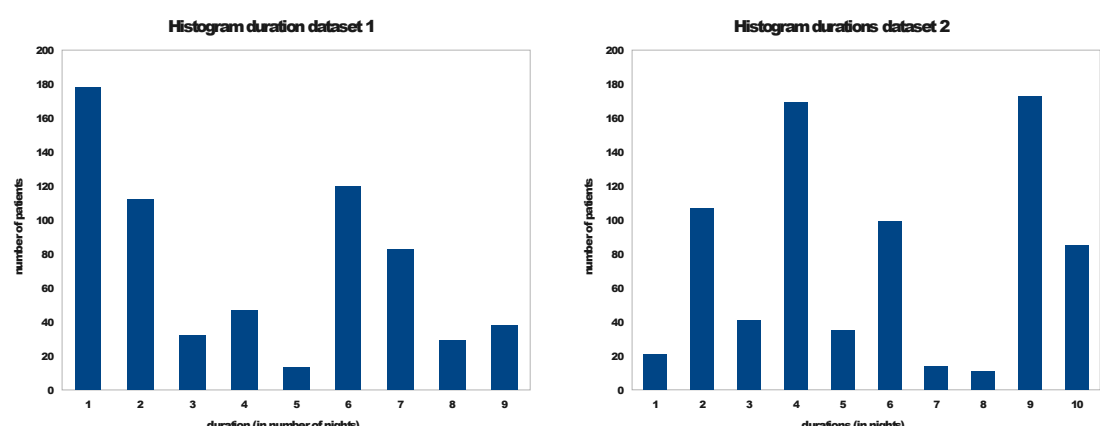

(a) Histogram of the durations in dataset 1 (b) Histogram of the durations in dataset 2
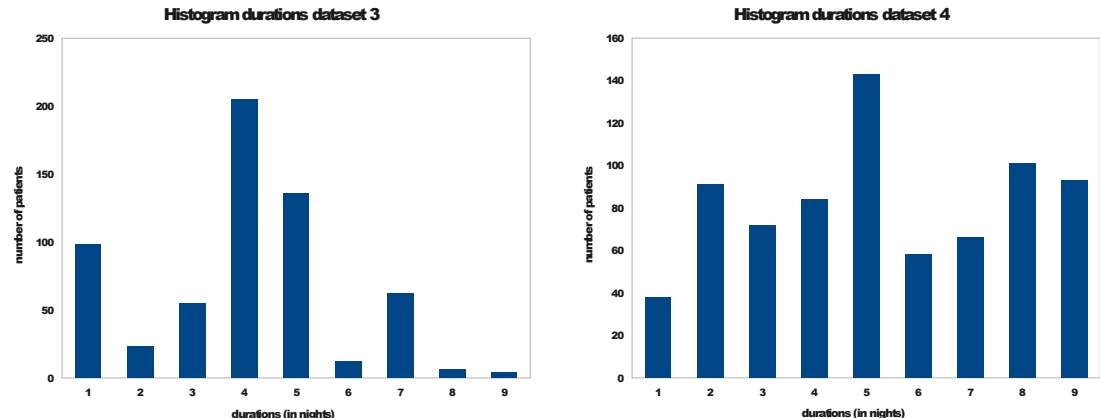

(c) Histogram of the durations in dataset 3 (d) Histogram of the durations in dataset 4
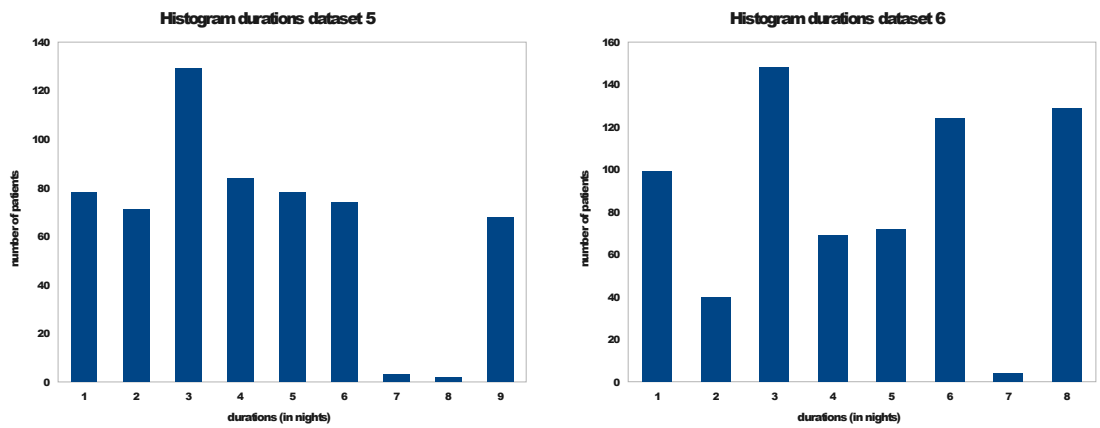

(e) Histogram of the durations in dataset 5 (f) Histogram of the durations in dataset 6

Fig. 2 Histogram of length of stays for every instance.

\subsection{Nurse Rostering Competition}

In the Nurse Rostering Competition two types of data instances are distinguished, called sprint, and medium. The names refer to the available computation time for the particular data instance types. As in the International Timetabling Competition, the organizers of the Nurse Rostering Competition have provided a benchmark too ${ }^{2}$

\footnotetext{
2 http://www.kuleuven-kortrijk.be/nrpcompetition/benchmarking
} 
that decides based on the specifications of the user's PC what the maximum allowed computation time per data instance type is.

- The simplest type of data instances (sprint) consists of 10 nurses. For these instances the computation time is on an average desktop PC about 10 seconds.

- The second type of data instances (medium) consists of around 30 nurses. The computation time is on an average desktop PC about 10 minutes.

Due to the inherent randomness of the approach, each solution method is run 10 times for every benchmark instance.

\subsection{Results}

\subsubsection{Patient admission scheduling}

The minimum, average, and standard deviation of the objective function over 10 runs for each benchmark instance are presented in Tables 3,4 and 5 The results of the experiments have been statistically processed for detecting significant differences with the Wilcoxon test. The best solution methods for each benchmark instance are indicated in bold. We call the set of solutions that perform statistically significant better than the others the best performing group. The hyperheuristics in Tables 3 , 4, and 5 are first ranked according to the number of times their results are in the best performing group. If there is a tie, the hyperheuristics are further ordered according to the best total average value over all instances.

For the patient admission scheduling problem the results show that the DHS heuristic selection mechanism combined with the great deluge move acceptance criterion and tournament factor 4 is the best performing hyperheuristic. There is however no statistical evidence that this combination ( $D H S-G D-4)$ performs statistically significant better than $S R-G D-16, C F-G D-4, D H S-G D-64$, and $S R-G D-4$. From the results it is clear that the hyperheuristics based on the great deluge move acceptance criterion are the best performers. The only improving move acceptance criterion results in the worst performing hyperheuristics. There is no statistical evidence to conclude that one of the heuristic selection criteria outperforms the others. Actually, this shows that the performance of the hyperheuristic depends largely on the performance of the move acceptance criteria.

Analyzing for example the best solution obtained for benchmark instance 1 (with a cost equal to 672.80) in more detail reveals that all hard constraints are satisfied, and that only the room type preference soft constraint is violated. This is a consequence of the fact that the demand of single and double rooms exceeds the supply.

The tabu search method that was applied on testdata 0 in [1] is outperformed by the current hyperheuristics approach. Even the worst performing hyperheuristic approach (DHS-OI-64) still finds better solutions for testdata0 than the token-ring tabu search algorithm.

\subsubsection{Nurse rostering}

We first have tried to solve the nurse rostering data sets exactly with an ILP solver (CPLEX). The ILP solver was interrupted when the available computation time was consumed. In some occasions, that was before the algorithm found the optimal solution. 


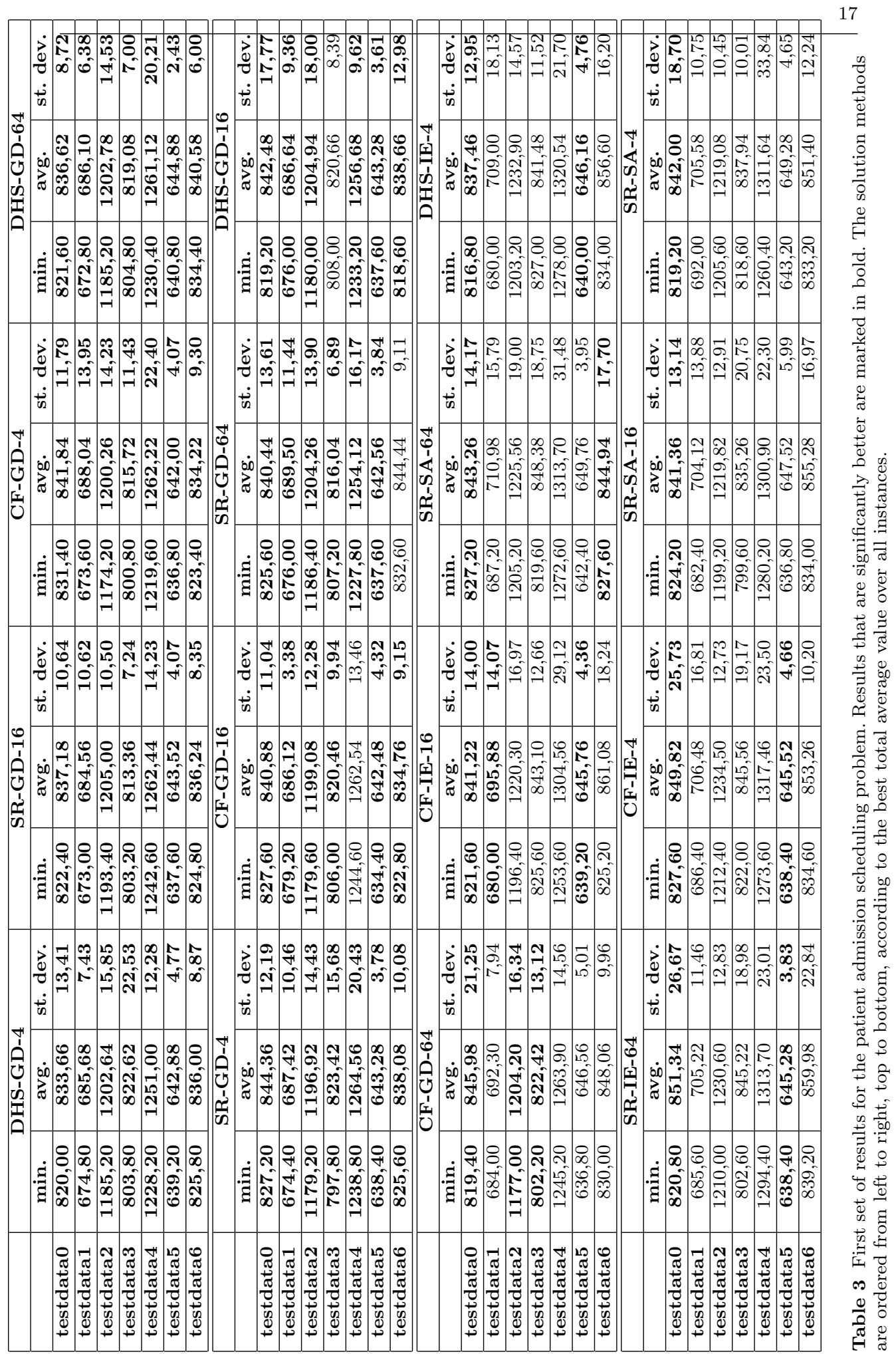




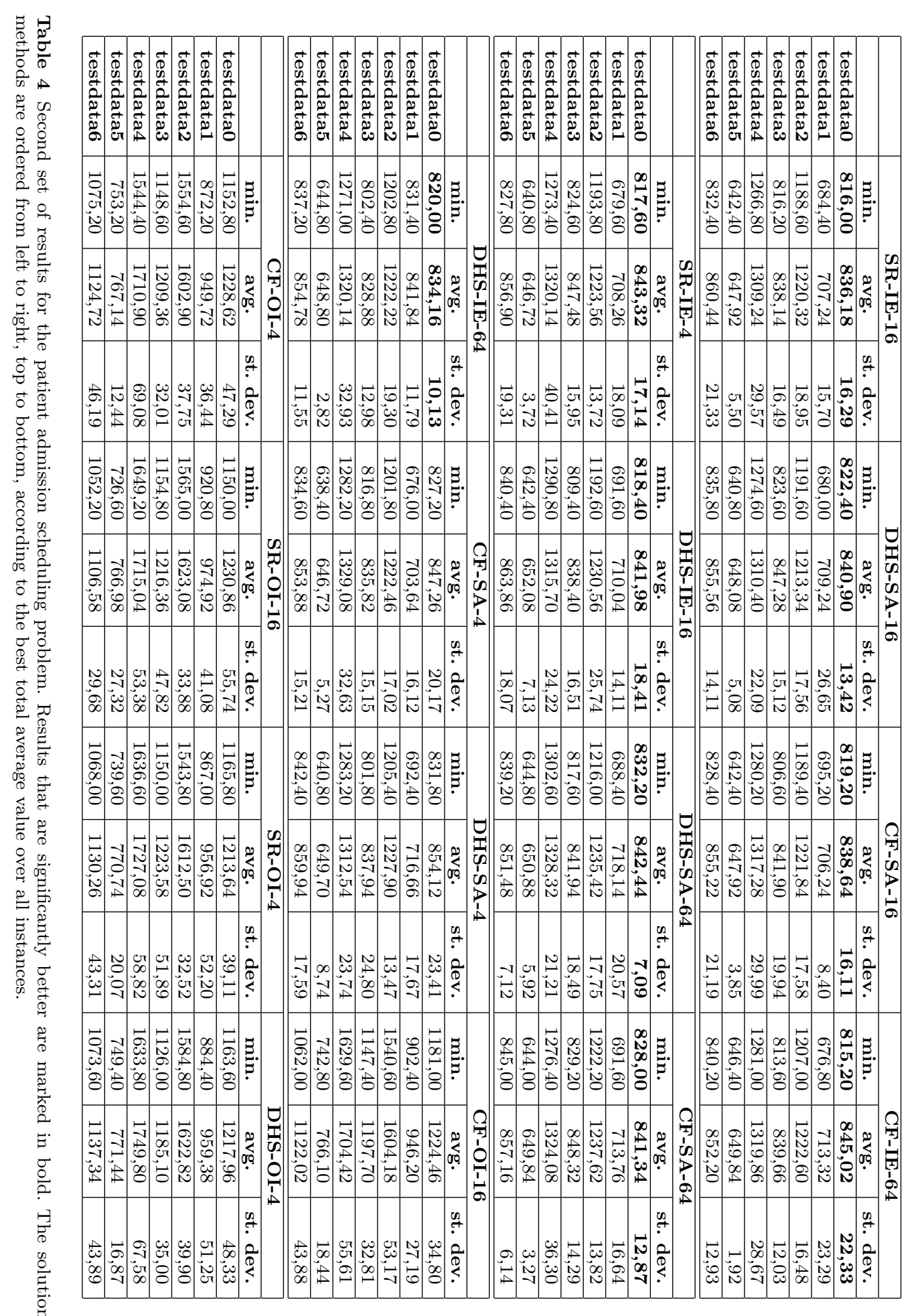




\begin{tabular}{|l|c|c|r|r|r|r|}
\hline & \multicolumn{3}{|c|}{ SR-OI-64 } & \multicolumn{3}{c|}{ DHS-OI-16 } \\
\hline & min. & avg. & st. dev. & min. & avg. & st. dev. \\
\hline testdata0 & 1174,80 & 1223,76 & 30,10 & 1135,40 & 1220,98 & 49,04 \\
\hline testdata1 & 937,00 & 987,12 & 35,26 & 888,20 & 982,72 & 60,02 \\
\hline testdata2 & 1583,60 & 1613,54 & 24,00 & 1519,80 & 1606,82 & 53,26 \\
\hline testdata3 & 1154,00 & 1208,54 & 41,85 & 1099,00 & 1204,88 & 56,08 \\
\hline testdata4 & 1595,40 & 1716,48 & 53,72 & 1642,80 & 1744,38 & 75,77 \\
\hline testdata5 & 739,20 & 768,16 & 19,18 & 746,40 & 768,56 & 17,70 \\
\hline testdata6 & 1090,80 & 1129,90 & 39,77 & 1108,20 & 1138,94 & 20,33 \\
\hline \hline & \multicolumn{7}{|c|}{ CF-OI-64 } & \multicolumn{3}{c|}{ DHS-OI-64 } \\
\hline & min. & avg. & st. dev. & min. & avg. & st. dev. \\
\hline testdata0 & 1140,20 & 1243,52 & 64,21 & 1179,00 & 1219,30 & 22,12 \\
\hline testdata1 & 892,00 & 951,16 & 36,27 & 946,60 & 992,80 & 29,26 \\
\hline testdata2 & 1532,20 & 1632,74 & 64,24 & 1550,80 & 1632,28 & 56,85 \\
\hline testdata3 & 1159,60 & 1198,52 & 31,33 & 1143,40 & 1209,32 & 41,64 \\
\hline testdata4 & 1660,40 & 1751,72 & 53,03 & 1664,80 & 1733,82 & 50,43 \\
\hline testdata5 & 743,20 & 771,44 & 19,10 & 754,00 & 788,04 & 21,43 \\
\hline testdata6 & 1063,20 & 1122,46 & 38,08 & 1068,80 & 1118,62 & 33,50 \\
\hline
\end{tabular}

Table 5 Last set of results for the patient admission scheduling problem. Results that are significantly better are marked in bold. The solution methods are ordered from left to right, top to bottom, according to the best total average value over all instances.

\begin{tabular}{|c|c|c|c|c|c|c|c|c|c|c|c|c|}
\hline \multicolumn{5}{|c|}{ medium } & \multicolumn{3}{|c|}{ medium_hint } & \multicolumn{5}{|c|}{ medium_late } \\
\hline 1 & 2 & 3 & 4 & 5 & 1 & 2 & 3 & 1 & 2 & 3 & 4 & 5 \\
\hline 240 & 240 & 236 & 237 & 303 & 84 & 119 & 14750 & 179 & 59 & 32 & 48 & 147 \\
\hline \multicolumn{10}{|c|}{ sprint } & \multicolumn{3}{|c|}{ sprint_hint } \\
\hline 1 & 2 & 3 & 4 & 5 & 6 & 7 & 8 & 9 & 10 & 1 & 2 & 3 \\
\hline 56 & 58 & 51 & 59 & 58 & 54 & 56 & 56 & 55 & 52 & 74 & 43 & 63 \\
\hline \multicolumn{10}{|c|}{ sprint_late } & & & \\
\hline 1 & 2 & 3 & 4 & 5 & 6 & 7 & 8 & 9 & 10 & & & \\
\hline 39 & 43 & 54 & 124 & 45 & 42 & 42 & 27 & 28 & 43 & & & \\
\hline
\end{tabular}

Table 6 ILP solutions for the nurse rostering problem data set. Values indicated in bold are optimal values.

This means that the optimal solution could not be obtained for all data sets. The results obtained with the ILP solver are presented in Table 6. Values indicated in bold are optimal solutions.

As for the patient admission scheduling problem we also applied the hyperheuristic approach on these data sets. The eight best performing hyperheuristics ${ }^{3}$ are presented in Table 7 and 8 In contrast to the patient admission scheduling problem, there is no hyperheuristic that is in the best performing group for all data instances. The hyperheuristic that is ranked first $(C F-G D-4)$ is for 30 out of 36 instances in the best performing group. The predominance of the great deluge based hyperheuristics is not as big as it was the case in the patient admission scheduling problem. Again, the hyperheuristics based on the only improving move acceptance criterion lead to the worst results. Also, there is no statical evidence that one of heuristic selection mechanisms outperforms the others.

\footnotetext{
3 The results for all 36 hyperheuristic variants can be found at http://allserv.kahosl.be/ peter/pas/JOH/results-nrc.xls
} 


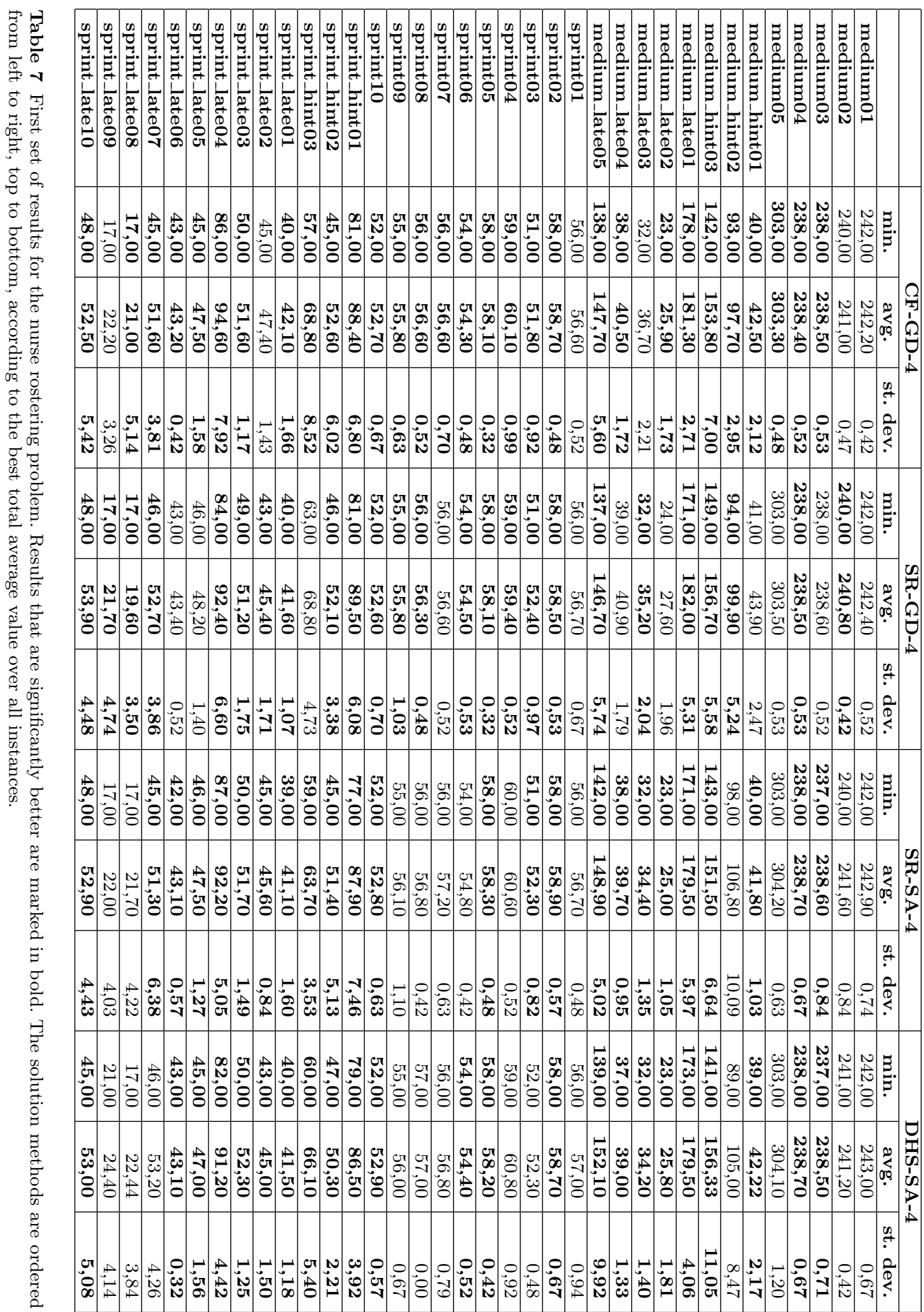




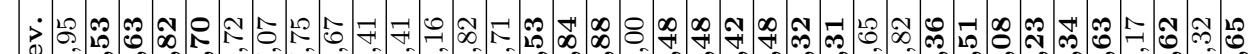
(i)

1. 党

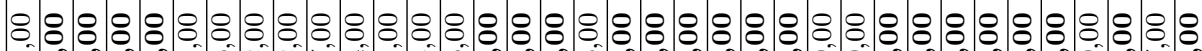
至

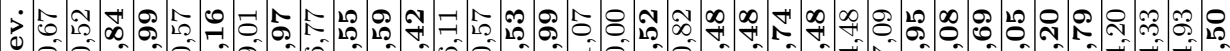

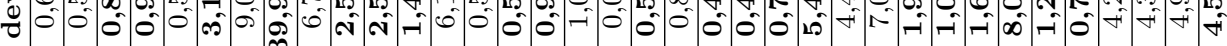
$\dot{\vec{n}}$

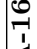
1.

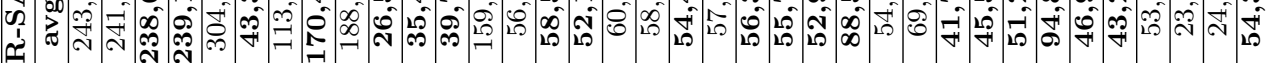
Uี

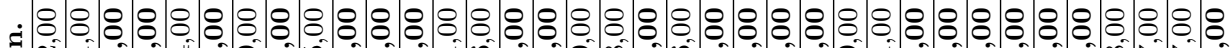
至

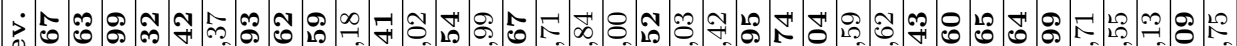

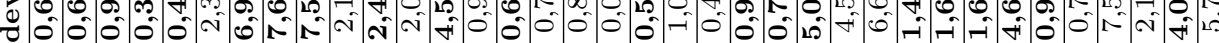
$+\dot{\vec{n}}$

V1

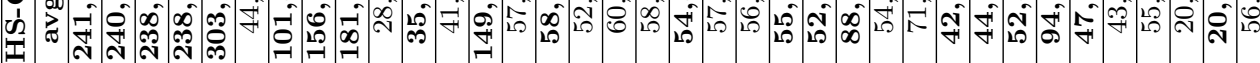
ค

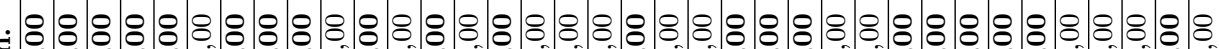

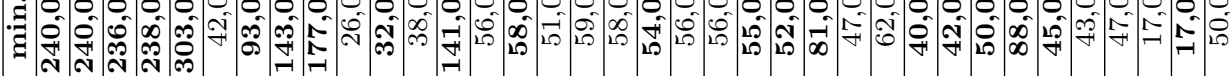

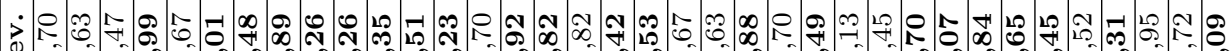

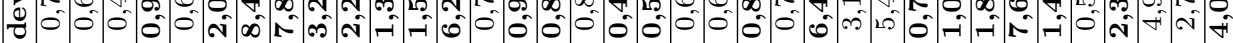
宛

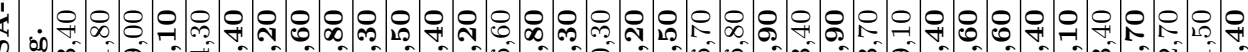

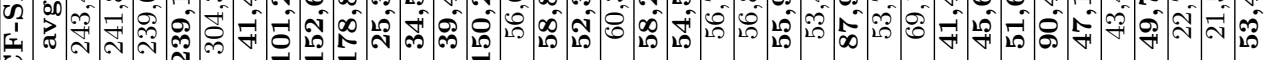
U

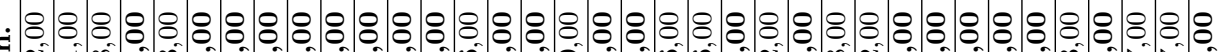

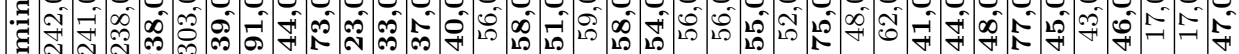

\section{Dit}

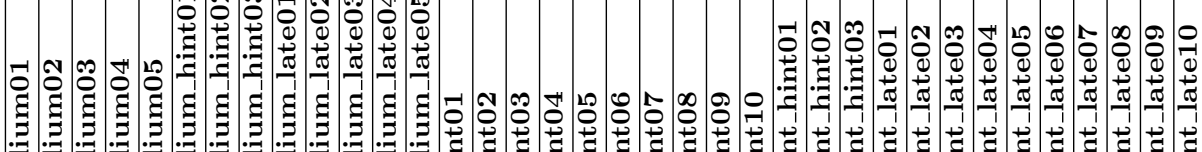

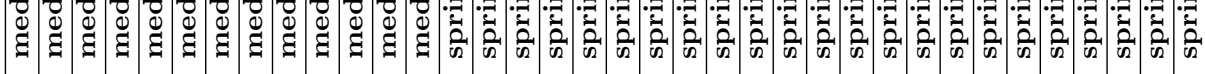


Bilgin et al. 3] conclude after numerous experiments on benchmarks with different combinations of move acceptance criteria and heuristic selection methods that there is not any combination of acceptance criteria and selection methods that dominates any other combination on all benchmarks. In their experiments, improving equal resulted in the best average performance, while choice function was on average slightly better than the other heuristic selection mechanisms. Our experiments show that the great deluge move acceptance criterion with tournament size 4 results in the best average performance. However, the heuristic selection mechanism is in both occasions different.

Although simple random is the easiest heuristic selection mechanism, it does not perform worse than the more 'intelligent' selection mechanisms. It is always good to compare as a first test a newly developed heuristic selection method with simple random.

\section{Conclusion}

We have introduced a general hyperheuristic approach for solving two operation research problems in health care. Regarding the first problem, the patient admission scheduling problem, we also provide the research community with new benchmark problems for the patient admission problem and a validation tool to compare algorithm performance. Other researchers are invited to address the problem instances and we will keep track of the best solutions on the Patient Admission Scheduling website 10. Within the problem settings of this paper, it is not possible to largely increase the bed occupancy rate in the hospital. That is due to the assumption of a fixed patient list and fixed patient stays over the considered time horizon. Assigning patients as much as possible to the room type of their choice increases their satisfaction during their stay. As a consequence it can also increase the income of the hospital, since, for example, Belgian doctors (can) demand a higher fee for treating patients assigned to a single room. The potential of the presented approach is to serve as an instrument for optimizing patient assignments each time new information becomes available (e.g. adjustment to the patient stay duration, additional patients on the list, canceled treatments, etc.) in a real world setting. It serves as an aid for the human admission scheduler to help him/her quickly decide to which room a patient should be assigned. Although not discussed in the paper, provisions have been made to fix patients that are already have been assigned and should not be moved during the search for a free bed for a new patient. The hyperheuristic approach significantly outperforms a tabu search algorithm that was previously applied to one of the instances.

Concerning the nurse rostering problem, we obtain with the same hyperheuristic approach results that are close to the solution obtained by an ILP approach in the same computation time. In fact, the hyperheuristic was first applied to the patient admission scheduling problem, and later adapted to tackle the nurse rostering problem. The only parts of the algorithm that were changed are the solution representation, the construction of the initial solution and the low-level heuristics. In a short software development cycle competitive results for the nurse rostering problem could be obtained. This paper shows that it is possible to obtain results in a 'good enough - soon enough - cheap enough' manner.

We advocate applying existing local search neighbourhoods, as heuristics within a hyperheuristic framework for general application. The implementation effort is limited compared to the experienced performance increase for the patient admission scheduling 
problem. As we have shown, the effect in performance is not limited to one problem only, and one can expect that this will be the case for different problems.

Directions for future research include 1) expanding the model for intensive care and day clinic units, adding extra real world constraints such as age compatibility, quarantine, and workload balance of the health care professionals, 2) developing new stochastic benchmark instances taking into account patients on waiting lists, 3) dynamic rescheduling in case of small changes to the data, and 4) tackling both problems as one integrated problem. The latter future research proposition can be clarified by the following reflection: the number of nurses needed per shift (the coverage) depends on the occupancy of the beds. The more patients that are assigned to beds, the more nurses will be needed. Both problems are actually intertwined.

\section{References}

1. M. Ayob and G. Kendall. A monte carlo hyper-heuristic to optimise component placement sequencing for multi head placement machine. In The International Conference on Intelligent Technologies, InTech'03, 2003.

2. B. Bilgin, P. De Causmaecker, and G. Vanden Berghe. A hyperheuristic approach to belgian nurse rostering problems. In Proceedings of the 4 th Multidisciplinary International Conference on Scheduling: Theory and Applications, pages 693-695, August 2009.

3. B. Bilgin, E. Özcan, and E. E. Korkmaz. An experimental study on hyper-heuristics and exam timetabling. In E. K. Burke and H. Rudová, editors, Revised Selected Papers of 6th International Conference on Practice and Theory of Automated Timetabling VI (Patat 2006), volume 3867 of $L N C S$, pages 394-412. Springer-Verlag, 2007.

4. E. K. Burke, T. Curtois, R. Qu, and G. Vanden Berghe. A scatter search approach to the nurse rostering problem. Journal of the Operational Research Society, page 25p, to appear.

5. E. K. Burke, M. Hyde, and G. Kendall. Evolving bin packing heuristics with genetic programming. In In proceedings of the 9th international conference on Parallel Problem Solving from Nature, volume 4193 of LNCS, pages 860-869, Reykjavik, Iceland, September 2006 2006. Springer.

6. E. K. Burke, G. Kendall, J. Newall, E. Hart, P. Ross, and S. Schulenburg. Handbook of Metaheuristics, chapter 16: Hyper-Heuristics: An Emerging Direction in Modern Search Technology, pages 457-474. Springer New York, 2003.

7. E. K. Burke, G. Kendall, and E. Soubeiga. A tabu-search hyperheuristic for timetabling and rostering. Journal of Heuristics, 9(6):451-470, 2003.

8. C.-F. Chien, F.-P. Tseng, and C.-H. Chen. An evolutionary approach to rehabilitation patient scheduling: A case study. European Journal of Operational Research, 189:12341253, 2008.

9. P. Cowling, G. Kendall, and E. Soubeiga. A hyperheuristic approach to scheduling a sales summit. In E.K. Burke and W. Erben, editors, Proceedings of the 3rd International Conference on Practice and Theory of Automated Timetabling, volume 2079 of LNCS, pages 176-190. Springer-Verlag Berlin Heidelberg, 2001.

10. P. Demeester, B. Bilgin, and G. Vanden Berghe. Patient admission scheduling benchmark instances. http://allserv.kahosl.be/ peter/pas/index.html, 2008.

11. P. Demeester, W. Souffriau, P. De Causmaecker, and G. Vanden Berghe. A hybrid tabu search algorithm for automatically assigning patients to beds. Artificial Intelligence in Medicine, 48(1):61-70, January 2010.

12. K. A. Dowsland, E. Soubeiga, and E. K. Burke. A simulated annealing based hyperheuristic for determining shipper sizes for storage and transportation. European Journal of Operational Research, 179(3):759-774, 2007.

13. G. Dueck. New optimisation heuristics. the great deluge algorithm and record-to-record travel. Journal of Computational Physics, 104:86-92, 1993.

14. P. Gemmel and R. Van Dierdonck. Admission scheduling in acute care hospitals: does the practice fit with the theory? International Journal of Operations and Production Management, 19(9):863-878, 1999. 
15. E. W. Hans, G. Wullink, M. van Houdenhoven, and G. Kazemier. Robust surgery loading. European Journal of Operational Research, 185:1038-1050, 2008.

16. S. Haspeslagh, P. De Causmaecker, M. Stølevik, and A. Schaerf. First international nurse rostering competition 2010. Technical report, K.U. Leuven, CODeS, May 2010.

17. A. K. Hutzschenreuter, P. A. N. Bosman, I. Blonk-Altena, J. van Aarle, and J. A. La Poutré. Agent-based patient admission scheduling in hospitals. In J.P. Mueller L. Padgham, D.C. Parkes and S. Parsons, editors, Proceedings of the Seventh International Conference on Autonomous Agents and Multiagent Systems - AAMAS-2008, pages 45-54. ACM, 2008

18. G. Kendall and M. Mohamad. Channel assignment in cellular communication using a great deluge hyper-heuristic. In Proc. of the 2004 IEEE International Conference on Network (ICON2004), 2004.

19. S. Kirkpatrick, C. D. Gelatt, and M. P. Vecchi. Optimization by simulated annealing Science, New Series, 220(4598):671-680, May 1983.

20. C. C. Marinagi, C. D. Spyropoulos, C. Papatheodorou, and S. Kokkotos. Continual planning and scheduling for managing patient tests in hospital laboratories. Artificial Intelligence in Medicine, 20:139-154, 2000.

21. M. Misir, K. Verbeeck, P. De Causmaecker, and G. Vanden Berghe. Hyper-heuristics with a dynamic heuristic set for the home care scheduling problem. In Proceedings of the IEEE Congress on Evolutionary Computation (CEC'10), Barcelona, Spain, July 18-23 2010

22. S. N. Ogulata and R. Erol. A hierarchical multiple criteria mathematical programming approach for scheduling general surgery operations in large hospitals. Journal of Medical Systems, 27(3):259-270, 2003

23. E. Özcan, B. Bilgin, and E. E. Korkmaz. A comprehensive analysis of hyper-heuristics. Intelligent Data Analysis, 12(1):3-23, 2008.

24. V. L. Smith-Daniels, S. B. Schweikhart, and D. E. Smith-Daniels. Capacity management in health care services: Review and future research directions. Decision Sciences, 19(4):889919, Fall 1988.

25. E. Soubeiga. Development and application of hyperheuristics to personnel scheduling. $\mathrm{PhD}$ thesis, University of Nottingham, 2003.

26. I. B. Vermeulen, S. M. Bohte, S. G. Elkhuizen, P. J. M. Bakker, and J. A. La Poutré. Decentralized online scheduling of combination-appointments in hospitals. In Proc. of the International Conference on Automated Planning and Scheduling. ACM, 2008.

27. I. B. Vermeulen, S. M. Bohte, S. G. Elkhuizen, H. Lameris, P. J. M. Bakker, and J. A. La Poutré. Adaptive resource allocation for efficient patient scheduling. Artificial Intelligence in Medicine, to appear.

28. I. B. Vermeulen, S. M. Bohte, D. J. A. Somefun, and J. A. La Poutré. Multi-agent pareto appointment exchanging in hospital patient scheduling. Service Oriented Computing and Applications, 1(3):185-196, November 2007.

\section{Appendix}

\subsection{Mathematical model}

The ILP model consists of the objective function to be minimized, the hard and soft constraints, the decision variables and the auxiliary variables. Let $N$ be the set of all nurses, $D$ the set of all days in the schedule period, and $S$ the set of all shift types.

The Decision Variables

$$
x_{n, d, s}=\left\{\begin{array}{l}
1 \text { if nurse } n \text { is assigned on day d and shift type } s \\
0 \text { otherwise }
\end{array}\right.
$$

\section{The Auxiliary Variables}

Let $|S|$ be the number of shift types.

$$
-|S| p_{n, d}+\sum_{j} x_{n, d, j} \leq 0
$$




$$
-p_{n, d}+\sum_{j} x_{n, d, j} \geq 0
$$

Equations 9 and 10 imply equation 11

$$
p_{n, d}=\left\{\begin{array}{l}
1 \text { if nurse } n \text { is assigned on day } d \\
0 \text { otherwise }
\end{array}\right.
$$

\section{Hard Constraints}

- Coverage constraint. Let $c_{d, s}$ be the number of assignments required on day $d$ and shift type $s$.

$$
\forall d \in D, \forall s \in S, \sum_{n} x_{n, d, s}=c_{d, s}
$$

- Single assignment per day.

$$
\forall n \in N, \forall d \in D \sum_{s} x_{n, d, s} \leq 1
$$

\section{Soft Constraints}

- Maximum days worked. Let $M$ be the constraint parameter, the maximum number of days a nurse is allowed to work.

$v_{n}$ denotes the number of violations to this constraint for nurse $n$.

$$
\forall n \in N, v_{n} \geq \sum_{d} \sum_{s} x_{n, d, s}-M
$$

The total number of violations to this constraint is calculated with equation 15

$$
T M a x=\sum_{n} v_{n}
$$

- Minimum days worked. Let $M$ be the constraint parameter, the minimum number of days a nurse is allowed to work.

$v_{n}$ denotes the number of violations to this constraint for nurse $n$.

$$
\forall n \in N,-v_{n}+\sum_{d} \sum_{s} x_{n, d, s} \geq M
$$

The total number of violations to this constraint is calculated with equation 17

$$
\text { TMin }=\sum_{n} v_{n}
$$

\section{- Unwanted Patterns}

Let $N=\{n \mid$ days where the pattern entry is "None" $\}$.

Let $A=\{a \mid$ days where the pattern entry is "Any" $\}$.

Let $S=\{s \mid$ shift types where a pattern entry is defined $\}$.

Let $D_{s}=\{d \mid$ days where a pattern entry is the shift type with the index $s\}$. 


$$
v+\sum_{n \in N} p_{e, n}-\sum_{a \in A} p_{e, a}-\sum_{s \in S} \sum_{d \in D_{s}} x_{e, d, s} \geq 1-|A|-\sum_{s \in S}\left|D_{s}\right|
$$

Equation 18 implies equation 19

$$
v=\left\{\begin{array}{l}
1 \text { if the assignment sequence satisfies the pattern. } \\
0 \text { otherwise }
\end{array}\right.
$$

- Complete identical weekends. Let $W$ be the set of all Saturdays in the schedule period.

$v_{n, d, s, 1}$ and $v_{n, d, s, 2}$ denote the number of violations to this constraint for nurse $n$, the weekend that starts with day $d$, and shift type $s$.

$$
\begin{aligned}
& \forall n \in N, \forall d \in W, \forall s \in S, v_{n, d, s, 1}-x_{n, d, s}+x_{n, d+1, s} \geq 0 \\
& \forall n \in N, \forall d \in W, \forall s \in S, v_{n, d, s, 2}+x_{n, d, s}-x_{n, d+1, s} \geq 0
\end{aligned}
$$

The total number of violations to this constraint is calculated with equation 22

$$
C I W=\sum_{n} \sum_{d} \sum_{s} v_{n, d, s, 1}+v_{n, d, s, 2}
$$

- The succession of shift types. Let $S^{\prime}$ be the set of shift type pairs $\left(s_{k}, s_{l}\right)$ such that $s_{l}$ is not allowed to be assigned the day after $s_{k}$ is assigned.

Let $D^{\prime}=\{d|d \geq 1 \wedge d<| D \mid\}$.

$v_{n, d,\left(s_{k}, s_{l}\right)}$ denotes the number of violations to this constraint for nurse $n$, day $d$, and shift type succession $\left(s_{k}, s_{l}\right)$.

$$
\forall n \in N, \forall d \in D^{\prime}, \forall\left(s_{k}, s_{l}\right) \in S^{\prime},-v_{n, d,\left(s_{k}, s_{l}\right)}+x_{n, d, s_{k}}+x_{n, d+1, s_{l}} \leq 1
$$

The total number of violations to this constraint is calculated with equation 24

$$
S N A=\sum_{n} \sum_{d} \sum_{\left(s_{k}, s_{l}\right)} v_{n, d,\left(s_{k}, s_{l}\right)}
$$

- Maximum consecutive working days. Let $M$ be the constraint parameter, the maximum number of consecutive working days for a nurse.

Let $D^{\prime}=\{d|d \geq 1 \wedge d \leq| D \mid-M\}$.

$v_{n, d}$ denotes the number of violations to this constraint for nurse $n$, and the day series that starts at day $d$.

$$
\forall n \in N, \forall d \in D^{\prime},-v_{n, d}+\sum_{k=0}^{M} p_{n, d+k} \leq M
$$

The total number of violations to this constraint is calculated with equation 26

$$
\text { MaxCon }=\sum_{n} \sum_{d} v_{n, d}
$$


- Minimum consecutive working days. Let $|M|$ be the constraint parameter, the minimum number of consecutive working days for a nurse.

Let $D^{\prime}=\{d|d \geq 1 \wedge d \leq| D \mid-M\}$.

Let $L=\{l \mid l \geq 2 \wedge l \leq M\}$.

$v_{n, d, l}$ denotes the number of violations to this constraint for nurse $n$, and the period between days $d$ and $d+l . v_{n, 0, l}$ denotes the number of violations to this constraint for nurse $n$, and the period between schedule period start and day $l$.

$$
\begin{gathered}
\forall n \in N, \forall d \in D^{\prime}, \forall l \in L,-\frac{v_{n, d, l}}{M-l+1}+p_{n, d}-p_{n, d+1}+p_{n, d+l} \geq 0 \\
\forall n \in N, \forall l \in L,-\frac{v_{n, 0, l}}{M-l+1}-p_{n, 1}+p_{n, l} \geq 0
\end{gathered}
$$

The total number of violations to this constraint is calculated with equation 29

$$
\text { MinCon }=\sum_{n} \sum_{l} v_{n, 0, l}+\sum_{n} \sum_{d} \sum_{l} v_{n, d, l}
$$

- Maximum consecutive free days. Let $|M|$ be the constraint parameter, the maximum number of consecutive free days for a nurse.

Let $D^{\prime}=\{d|d \geq 1 \wedge d \leq| D \mid-M\}$.

$v_{n, d}$ denotes the number of violations to this constraint for nurse $n$, and the day series that starts at day $d$.

$$
\forall n \in N, \forall d \in D^{\prime}, v_{n, d}+\sum_{j=0}^{M} \sum_{s} x_{n, d+j, s} \geq 1
$$

The total number of violations to this constraint is calculated with equation 31

$$
\text { MaxFree }=\sum_{n} \sum_{d} v_{n, d}
$$

- Minimum consecutive free days.

Let $|M|$ be the constraint parameter, the minimum number of consecutive free days for a nurse.

Let $D=\{d|d \geq 1 \wedge d \leq| D \mid-m\}$.

Let $K=\{k \mid k \geq 2 \wedge k \leq M\}$.

$v_{n, d, k}$ denotes the number of violations to this constraint for nurse $n$, and the period between days $d$ and $d+k$. $v_{n, 0, k}$ denotes the number of violations to this constraint for nurse $n$, and the period between schedule period start and day $k$.

$$
\begin{gathered}
\forall n \in N, \forall d \in D, \forall k \in K, v_{n, d, k}-p_{n, d}+p_{n, d+1}-p_{n, d+k} \geq-1 \\
\forall n \in N, \forall k \in K, v_{n, 0, k}+p_{n, 1}-p_{n, k} \geq 0
\end{gathered}
$$

The total number of violations to this constraint is calculated with equation 34

$$
\text { MinFree }=\sum_{n} \sum_{k} v_{n, 0, k}+\sum_{n} \sum_{d} \sum_{k} v_{n, d, k}
$$


The Objective Function

The objective of the optimization is to minimize $F$, which equals to the number of all violations of the soft constraints (Equation 35).

$F=T M a x+T M i n+C I W+S N A+$ MaxCon + MinCon + MaxFree + MinFree $+S A D$ 